\title{
Psychotherapy with traumatised refugees - the design of a randomised clinical trial
}

\author{
Erik Vindbjerg, Cand.psych*, Christoph Klimpke, Cand.psych*, \\ Jessica Carlsson, MD, PhD*, **
}

\begin{abstract}
There is little evidence as to which kind of psychotherapy is the most effective in the treatment of traumatised refugees. At the Competence Center for Transcultural Psychiatry, a series of clinical trials have been conducted since 2008. The first results are pending publication. The aim of this paper is to discuss some of the challenges in adapting Cognitive Behavioural Therapy (CBT) to the treatment of traumatised refugees, as well as describe a randomised clinical trial designed to test two such adaptations. In the described trial one group receives CBT with a focus on cognitive restructuring while the other group receives CBT focusing on Stress Management. A main goal of this setup is to test whether some, perhaps even most, of the traumatised refugees referred to treatment, may benefit from a more direct focus on current stress, and its alleviation through simple, repetitive exercises, compared to a focus on analysing and changing thought patterns.
\end{abstract}

Keywords: Post-traumatic Stress Disorder, Depression, Psychotherapy

\footnotetext{
*) The Competence Center for Transcultural Psychiatry (CTP), Psychiatric Center Ballerup, the Mental Health Services of the Capital Region of Denmark

${ }^{\star}$ ) University of Copenhagen
}

Correspondence to: jessica.carlsson.lohmann@regionh.dk

\section{Introduction}

There is limited evidence as to which kind of psychotherapy is the most effective in the treatment of traumatised refugees. ${ }^{1,2}$ Regarding the treatment of other clinical populations with PTSD, such as war veterans or rape victims, much more empirical support has been accumulated. ${ }^{3,4} \mathrm{~A}$ Cochrane review from 2009 on the treatment effect of psychotherapy on PTSD concludes that there seems to be an effect of Cognitive Behavioural Therapy CBT, especially Trauma Focused CBT (TF-CBT). ${ }^{1}$ Two other methods showing promising results are Eye Movement Desensitization and Reprocessing (EMDR) and Stress Management. ${ }^{1}$ For the refugee population with PTSD, reviews point to a possible effect of CBT and Narrative Exposure Therapy (NET).$^{5-7}$ Of the randomised trials incorporating CBT, all but one have been conducted with Indochinese refugees, using a culturally adapted version of CBT. ${ }^{8}$ Indeed CBT was thought to be adaptable to this population "owing to the similarity of Buddhist principles to core aspects of CBT." "Thus, for the most part, it remains to be shown whether CBT results can be replicated with other refugee populations.

The first randomised trial (Trial 1), conducted at the Competence Center for Transcultural Psychiatry (CTP) measured the effect of TF-CBT compared to pharmacotherapy in a mixed population of traumatised refugees (results are currently being 
analysed). The trial was intended as the first in a series, in an endeavour to improve the evidence base of treatments of traumatised refugees. As a unique feature, a basic infrastructure was designed, that allowed the integration of research and routine intervention. Based on the clinical experiences during Trial 1 , a subsequent randomised trial (Trial 2) was planned in which TF-CBT with a focus on cognitive restructuring is compared to Stress Management.

In this paper, we will:

a) describe the main challenges we have encountered so far when using TF-CBT with traumatised refugees

b) introduce the general setup of the Trial PTF2

c) discuss the two psychotherapy treatments of PTF2, which are designed using the clinical experiences from PTF1.

\section{Challenges using manualised TF-CBT}

During Trial 1 psychotherapy followed a manual based on mainly TF-CBT with added elements of mindfulness, and Acceptance and Commitment Therapy. ${ }^{9}$ Because of the dominance of TF-CBT in Trial 1, and the evidence for the effect of TF-CBT in PTSD we will focus the discussion below on TF-CBT. The same group of psychologists have been involved in authoring and using the manual, and subsequently, on an ongoing basis, evaluating its implementation. After conducting the trial, three overall, clinical challenges stand out when using the manualised TF-CBT:

\section{Communication}

Refugees may have very different concepts of psychological distress, and simply disclosing and separating thoughts, feelings, bodily sensations and behaviours can be challenging. Using interpreters makes this even more time consuming and challenging, as does the fact that many traumatised refugees will initially find it hard to trust the therapist and/or the interpreter. So, although TF-CBT offers a new and potentially more adaptive way of thinking about psychological distress, it is also a very time-consuming investment.

\section{Trauma-focus}

Most of our patients carry several traumatic memories, yet complain as much about problems related to depression and post migration stress, e.g. feelings of hopelessness and worrying about letters from the authorities, as compared to flashbacks or specific, recurrent nightmares. Hypervigilance and avoidance are very predominant, and a clear testament to past traumatic experiences. But, they are also intensified if patients, as a desperate measure, perhaps for years, have turned away from the overwhelming stimulation of social contact and physical activity. Such behaviour clearly takes its toll on the capacity for emotion regulation and the ability to communicate openly with others. Accordingly, with patients in this state, we have often found it overly ambitious to extensively work through past traumas.

\section{Manualisation}

The manual used in Trial 1 suggested a rather strict, consecutive succession of themes and techniques. This has been, and to some degree still is, a widely used way to standardise treatment in randomised psychotherapy studies. However, with traumatised refugees often presenting a wide palette of comorbidities, such as depression, pain, psychosomatic symptoms and changes of personality, as well as social problems and well founded worries about family members left behind, a rather flexible manual is called for.

The challenges outlined above may be overcome in different ways for each individual 
patient. For instance, communication will undoubtedly improve when the therapist can adapt to the unique conceptual framework of each culture, e.g. culture-specific idioms of distress. ${ }^{11}$ The focus in this article is to balance such concerns with the need for some measure of standardisation, so as to allow for a randomised trial. Therefore, what follows is in no way intended as an exhaustive solution to said challenges. Rather, it is a solution that, if implemented in a flexible manner, may both be effective and provably so.

\section{The design of the present randomised trial}

The new randomised trial, Trial 2, was designed during the last part of Trial 1 and implemented directly following it. The design was not based on outcome data, which was only available later, but rather the clinical experiences of the therapists and feedback from the patients.

Stress Management (SM) was chosen as the focus of Trial 2 for a number of reasons. First of all, SM has already obtained empirical support with other traumatised populations. ${ }^{1}$ Second, SM offers potential solutions to the challenges outlined above, in that it a) appears easy to communicate, $b$ ) focuses on current, discrete problems rather than past traumas, and c) allows for a flexible manual. Focusing on relaxation, attention-diversion and behavioural activation, this manual would help patients build a better capacity to deal with stress. Furthermore, the causal mechanism would be conceptualised as a "stress vaccine": by breaking a cycle of avoidance, e.g. of other people and of physical activity, and aided by basic stress reduction techniques, the patient would build up an inherent capacity for emotional regulation, akin to boosting an immune system. We believed that this analogy would be easy to communicate to the patient, would allow the patients to better understand the rationale of the individual interventions, and hence promote motivation, and lastly, allow us to implement a rather standardised treatment.

As the alternative treatment in Trial 2 we chose to offer TF-CBT with a focus on assessing and changing cognitions. The aims of the treatment would be to 1) help patients understand how negative, automatic thoughts influence body, feelings and behaviour, 2) to make patients capable of identifying inappropriate and stressful thought patterns in their own lives, and 3) to replace these thought patterns with more realistic and less destructive thought patterns. Because of this focus, we have named this condition "cognitive restructuring”. By nature of being trauma focused, TF-CBT will still try to tackle the challenge of working through traumas directly, whereas SM will mostly bypass this and focus on current problems. As for the other challenges of easy communication and a flexible manual, we have strived to optimise the TF-CBT manual, while still sticking to the same core concept of analysing thoughts and behaviour.

To test and compare the two treatments we set up a randomised study (PTF2). In the following we will describe the general study design and end with the description of the two manuals.

\section{Population}

Medical doctors carried out the pre-treatment assessments at CTP. All patients fulfilling the inclusion criteria (see table 1) were invited to participate in the research study at the pretreatment assessment. Patients who did not wish to participate were offered a treatment of the same extent, with methods from any of the three manuals, and with less monitoring. The inclusion and exclusion criteria in PTF2 are described in Table 1. A total of 140 out of 189 patients were included and in April 2013 the last 


\section{Table 1}

\section{Inclusion criteria}

- Admitted to treatment at CTP 15 June 2011-31 March 2012

- $>=18$ years

- Being a refugee /or having been family reunified with a refugee

- Symptoms of PTSD according to the International Classification of Diseases-10 (ICD-10 F 43.1)

- A history of trauma (typically imprisonment with torture, organised violence, persecution or war experiences)

- Referred by a general practitioner, psychiatric practitioner or medical doctor at a psychiatric hospital

- Being motivated for treatment

- Informed consent

\section{Exclusion criteria}

- Patients diagnosed with a psychotic disorder (ICD-10 F2x

- $\quad$ and F30.1-30.9)

- Alcohol or drug abuse at pretreatment assessment (ICD-10 F1x)

- Need for being admitted to a psychiatric ward at pretreatment assesment.

participants finished treatment. All participants were offered treatment with a medical doctor (a trainee in psychiatry or a psychiatrist) combined with psychotherapy provided by a psychologist. The length of treatment was approximately 6 months. At the time of inclusion participants were randomised to either CBT with a focus on Stress Management or CBT with a focus on Cognitive Restructuring. Both treatment modalities were offered by all psychologists, i.e. there was no systematic division of labour and expertise across the two types of treatment.

It should be noted, that only refugees that have already been granted asylum are offered treatment at CTP. Asylum seekers are offered treatment by the Red Cross in Denmark. Many of the refugees (or family members reunified with a refugee) referred to CTP have been through a lengthy asylum process.

\section{Data collection}

The ratings scales used were the Hopkins Symptom Check List -25 (HSCL-25), 12,13 the somatisation items from the SCL-90, the Harvard Trauma Questionnaire (HTQ), 14,15 the WHO- $5,{ }^{16}$ the Sheehan Disability Scale (SDS), ${ }^{17}$ the Global Assessment of Functioning, symptom and functioning scale (GAF-S \& GAF-F), ${ }^{18}$ the Hamilton Anxiety Rating Scale (Ham-A), ${ }^{19}$ the Hamilton Depression Rating Scale (Ham-D), ${ }^{20,21}$ pain on visual analogue scale and Goal Attainment Scaling (GAS). ${ }^{22}$ The rating scales and the frequency of the ratings are described briefly in Figure 1. At the end of treatment, the patient was interviewed using open-ended questions regarding their present health and their evaluation of the treatment.

\section{Setting and treatment}

The study was carried out at CTP. CTP is part of the Mental Health Services of the 
Figure 1: Overview of treatment and ratings

Questionnaires: (in Arabic, Farsi/Dari, Bosnian/Croatian/Serbian or Danish).

Harvard Trauma Questionnaire - a self-administered questionnaire used to measure symptoms of PTSD. ${ }^{13,14}$ The first 16 items in part IV of the HTQ are used. The 16 items cover all criteria in PTSD in both ICD-10 and the Diagnostic and Statistical Manual of Mental Disorders- IV (DSM-IV).

Hopkins Symptom Checklist 25 - a self-administered questionnaire measuring symptoms of anxiety and depression. ${ }^{11,12}$ The HSCL-25 was originally developed from Symptom Checklist -90 (SCL-90). HSCL-25 contains 25 items, 10 anxiety and 15 depression items. It has been proven a valuable instrument in many different cultures.

SCL-90, 12 somatisation items only

WHO-5 Well-being Index - a self-administered questionnaire regarding perceived quality of life. It contains 5 items. The questionnaire has been used to rate quality of life in different diagnostic groups in psychiatric patients. ${ }^{15}$

Sheehan Disability Scale - a self-administered questionnaire regarding level of functioning as to family, work and social relations using visual analogue scales (VAS). ${ }^{16}$

Pain is evaluated on a VAS.

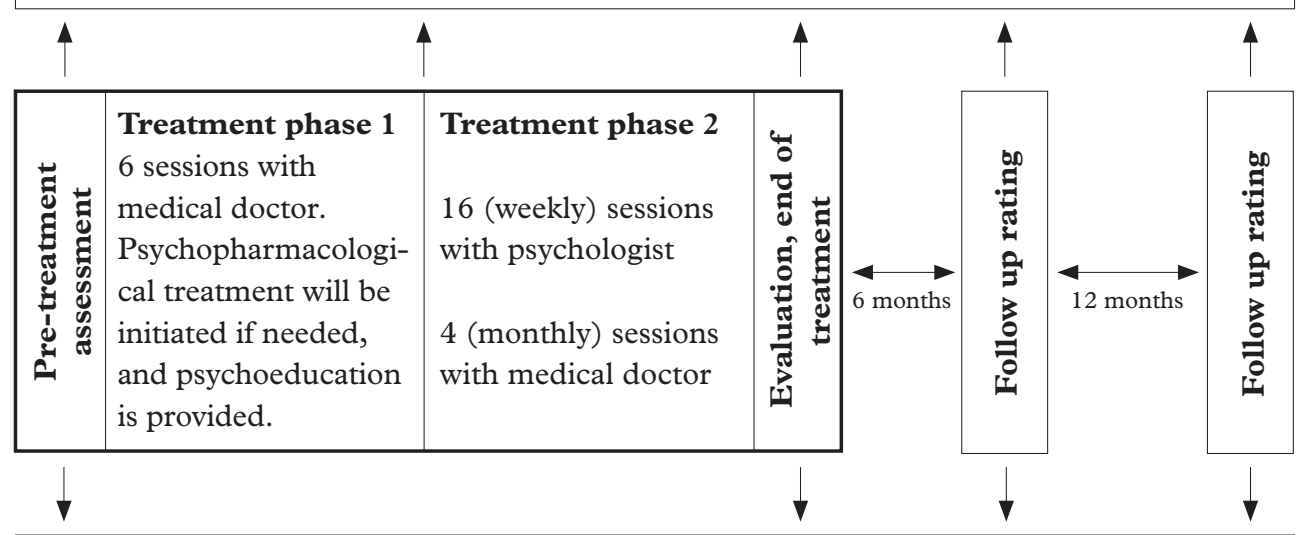

Hamilton Anxiety Rating Scale (Ham-A) (blinded) - an observation rating scale measuring the level of anxiety based on a semi-structured interview. The scale is extensively used in psychiatric research. ${ }^{18}$

Hamilton Depression Rating Scale (Ham-D) (blinded) - an observation rating scale measuring the level of depression based on a semi-structured interview. ${ }^{19,20}$ The Ham-D has been widely used in psychiatric research.

Global Assessment of Functioning - observation ratings used to rate the level of functioning in adults. ${ }^{17}$ The scales have been validated in a number of languages and are commonly used in clinical studies in psychiatry. 
Capital Region of Denmark. The staff at CTP consists of medical doctors (trainees in psychiatry and psychiatrists), psychologists and social workers. The treatment model used at CTP is presented in Figure 1.

\section{Ethical Considerations}

The study had been accepted by the Regional Committee for Ethics in Medical Science and the Danish Data Protection Agency. The procedures in the study are all in accordance with the Helsinki Declaration. The primary goal of CTP and the accompanying manuals is to contribute to a systematical evaluation of treatment in a field where, as of yet, very little evidence exists.

\section{The psychotherapeutic Manuals}

As mentioned before, each of the two treatment conditions were defined by their psychotherapy manual. These manuals will be described in more detail below.

\section{The Stress Management Manual}

Different programs have been built on the concept of Stress Management (SM). ${ }^{23}$ In the treatment of PTSD one of the most commonly used and evidence based of these programs is Stress Inoculation Training. According to this program, and following the view of Lazarus and Folkman, pathological stress is caused by an insufficient ability to cope with stress and anxiety, and the primary goal of the therapy, according$1 y$, is to help patients acquire and consolidate a number of coping skills. ${ }^{24,25}$ Thus, the sessions revolve around education, acquisition and application of these new coping skills. In the SM manual, such techniques are presented in three groupings: 1) relaxation, 2) attention diversion and 3) behavioural activation. Each of these groupings is further divided into three subgroups.

\section{Relaxation}

Relaxation consists of a combination of breathing exercises, body relaxation and visualisation exercises, in which the patient is taught to visualise a secure, trauma free zone. These exercises have been shown to be valuable in reducing the physiological tension which accumulates from stress. The effect size in previous studies has typically been found to be moderate, with interventions typically entailing weekly training sessions and daily home practice. ${ }^{26}$ The techniques have been used to reduce anxiety, including relational insecurity and performance anxiety, as well as to alleviate chronic muscle tension, headaches and pain in neck and back, insomnia and increased blood pressure. $^{26}$

\section{Attention-diversion}

Attention-diversion entails the shifting away of thoughts, feelings or impulses from an unwanted or uncomfortable state. Diversion consists of a conscious interruption of the ruminations, flashbacks and burdensome feelings which patients are often absorbed in. Diversion-techniques are used to consciously shift bleak thoughts, feelings or impulses towards other things so the patient is brought into contact with, and becomes aware, of present circumstances.

The first attention-diversion technique, thought-stop, aims for a short and intense interruption of unwanted thoughts and rumination. This can be achieved by various means and is often a question of 'what works for whom'. Among the techniques used are counting backwards from 100 to 0 as fast as possible or washing one's face with cold water. Outer focus as opposed to thought-stop aims at letting oneself get immersed in what happens around oneself and thus shifting one's attention away from mental and bodily processes, which tend to dominate attention 
in anxiety and depression. Finally, emotioncontrol techniques are introduced in order to find concrete coping-thoughts to be used in situations which are considered difficult by the individual patient, the aim being the control of stress and anxiety-reactions in everyday life. In preparation for such a difficult situation the patient is trained to visualise the situation, acknowledge the feelings that emerge in the situation and to use relaxation-techniques and copingthoughts to control it.

\section{Behavioural activation}

Posttraumatic stress, depression and anxiety initiate a vicious circle which tends to reduce the patient's cognitive and physical level of functioning. Most activities are experienced as a burden, hard to overcome, and easily bringing exhaustion. This vicious circle can be characterised in the following general terms: The less active one is, the less active one wants to be. Behavioural/activity-activation offers a number of techniques to break this vicious circle.

One technique in the domain of behavioural/activity-activation is visualisation, where the patient imagines himself conducting an activity he or she wishes to be able to master in real life. The rationale behind this is that if one is able to imagine oneself conducting a certain activity one is more likely to be able to execute this activity efficiently in real life.

The goal of another exercise, activityplanning, is for the patient to regain (at least some degree of) control over his or her life via the active planning and subsequent execution of activities which entail feelings of pleasure and coping/efficacy. Furthermore this technique can clarify how patients spend their time and contribute in challenging negative automatic thoughts like "I'm not doing anything at all" or "I can't manage anything". Working with such cognitions is still welcome in SM, but less time and focus is spent on eliciting these cognitions. And change is facilitated more by physical coping actions and the accompanying perception of control, rather than working with cognitions themselves, testing out different ways of thinking.

Finally, in evening-therapy the patient is encouraged to write down three positive experiences, incidents or thoughts that have occurred during the day in order to actively counteract the common tendency to focus on the negative aspects of life. This has also been shown to improve sleep in some patients. $^{27}$

\section{The manual for cognitive restructuring}

The cognitive restructuring (CR) manual consists mainly of psycho-education, cognitive restructuring of negative thoughts resulting from traumatic experiences, and exposure. The CR manual has been organised with a number of themes for the therapist to select from, based on a clinical evaluation and according to the capabilities and needs of the patient. Each theme consists of psycho-education, suggestions for intervention, as well as suggestions for homework assignments.

The first chapters introduce the patient to CBT, common trauma reactions, as well as depression and anxiety. The following chapters more specifically address how traumatic experiences can influence one's view of self, others and the world, as well as how they can hamper one's trust in others and increase the need for control. One chapter is dedicated to loss and grief, another chapter focuses on guilt, shame and self-blame, and yet another chapter instructs exposure either to bodily symptoms, to reliving of past trauma or through rehearsing future situations, in which the patient wishes 
to give up safety behaviours. The last chapter addresses relapse prevention, including "warning signals" and future high-risk situations, to be discussed at the end of therapy. Through these chapters, the CR manual allows us to work with cognitions, behaviour, feelings and bodily sensations, with cognitions as particularly important mediators.

\section{Methodological challenges}

Each manual, both the SM and CR, may appear ambitious in their own way. And indeed they were never intended to be exhausted in a single treatment course. At the same time, manual based psychotherapy will inevitably entail much more than is described in the manual. Treatment outcome will depend on personal as well as professional qualities of the therapist, the resources and motivation of the patient, and the working alliance. A good manual should not detract too much attention from these parts of therapy, which do not lend themselves so easily to standardisation. Yet a treatment manual, through revisions, may help us capture those interventions that do in fact work well across this diverse patient group.

With the current setup we hope to be able to offer a qualified (albeit not definitive) answer to, whether short term TF-CBT is feasible for treating traumatised refugees, or whether CBT with a focus on current stress offers a better match for most of this population. Also, we hope that the setup demonstrates an apt and relevant way to test such hypothesis with both qualitative observations and quantitative measures.

As mentioned earlier, TF-CBT was originally chosen for PTF1 due to its particularly strong evidence base in the treatment of PTSD. As described throughout this article, in PTF2 TF-CBT has been compared to Stress Management, a poten- tially more flexible and easily communicable therapy, which also shows evidence of effectiveness. Keeping these priorities in mind, and looking ahead, Narrative Exposure Therapy (NET) may be an interesting candidate for a future RCT-study. Firstly, NET has in recent years gained significant evidence in the specific field of traumatised refugees. Secondly, NET might offer alternative approaches to the three key challenges outlined in this article, i.e. trauma-focus, manualisation, and, perhaps most prominently, communication.

The collected data from the randomised study PTF2 will be analysed in 2014, and results will be reported in future articles. Hence, we have yet to see whether the intended improvement has had significant impact on treatment outcome. In this descriptive article, however, we have outlined some of the main challenges in implementing and adapting evidence-based treatments to refugee populations, as well as demonstrated a detailed setup for testing such adaptations.

\section{Acknowledgements}

The following psychologists have been involved in the development of the treatment manuals at CTP: Karin Riber, Mette Jensen, Kenan Hansen, Naderah Parwani, Eneli Kramer Hero, Dorthe Boye Hjortkjær, Ida Andersen, Juno Calmer.

This research study has been supported by TrygFonden.

References:

1. Bisson J, Andrew M. Psychological treatment of post-traumatic stress disorder (PTSD). Cochrane Database Syst Rev. 2007 Jul 18;(3):CD003388.

2. Jaranson JM, Quiroga J. Evaluating the services of torture rehabilitation programmes: history and recommendations. Torture. 2011;21(2):98-140. 
3. Foa EB, Rothbaum BO, Riggs DS, Murdock TB. Treatment of posttraumatic stress disorder in rape victims: A comparison between cognitivebehavioural procedures and counselling. J Consult Clin Psychol. 1991 Oct;59(5):715-23.

4. Schnurr PP, Lunney CA, Sengupta A, Waelde LC. A descriptive analysis of PTSD chronicity in Vietnam veterans. J Trauma Stress. 2003 Dec;16(6):545-53.

5. Crumlish N, O'Rourke K. A systematic review of treatments for post-traumtic stress disorder among refugees and asylum-seekers. J Nerv Ment Dis. 2010 Apr;198(4):237-51 .

6. Hinton DE, Chhean D, Pich V, Safren SA, Hofmann SG, Pollack MH. A randomized controlled trial of cognitive-behaviour therapy for Cambodian refugees with treatment-resistant PTSD and panic attacks: a cross-over design. J Trauma Stress. 2005 Dec;18(6):617-29 .

7. Neuner F, Kurreck S, Ruf M, Odenwald M, Elbert T, Schauer M. Can asylum-seekers with posttraumatic stress disorder be successfully treated? A randomized controlled pilot study. Cogn Behav Ther. 2010;39(2):81-91.

8. Palic S, Elklit A. Psychosocial treatment of posttraumatic stress disorder in adult refugees: a systematic review of prospective treatment outcome studies and a critique. J Affect Disord. 2011 Jun;131(1-3):8-23.

9. Hjortkjær DB, Hero EK, Calmer J, Hansen K, Jensen M, Parwani N. Psykoterapi med traumatiserede flygtninge. Psykolognyt. 2009;10:12-6. Available from: http://infolink2003.elbo.dk/ PsyNyt/Dokumenter/doc/15905.pdf

10. Kirmayer, LJ. Cultural variations in the clinical presentation of depression and anxiety: implications for diagnosis and treatment. J Clin Psychiatry. 2001;62 suppl 13:22-8; discussion 29-30.

11. Derogatis LR, Lipman RS, Rickels K, Uhlenhuth EH, Covi L. The Hopkins Symptom Checklist (HSCL): A self-report symptom inventory. Behav Sci. 1974 Jan;19(1):1-15.

12. Mollica RF, Wyshak G, de Marneffe D, Khuon F, Lavelle J. Indochinese versions of the Hopkins Symptom Checklist-25: a screening instrument for the psychiatric care of refugees. Am J Psychiatry. 1987 Apr;144(4):497-500.

13. Hollifield M, Warner TD, Lian N, Krakow B, Jenkins JH, Kesler J, et al. Measuring trauma and health status in refugees: a critical review. JAMA. 2002 Aug 7;288(5):611-21.

14. Mollica RF, Caspi-Yavin Y, Bollini P, Truong T, Tor S, Lavelle J. The Harvard Trauma Questionnaire. Validating a cross-cultural instrument for measuring torture, trauma, and posttraumatic stress disorder in Indochinese refugees. J Nerv Ment Dis. 1992 Feb;180(2):111-6.

15. Folker H, Folker AP. WHO-5 as a simple method for measuring quality of life in daily psychiatric clinics. Ugeskr for Læger. 2008 Mar 3;170(10):830-4.

16. Sheehan KH, Sheehan DV. Assessing treatment effects in clinical trials with the discan metric of the Sheehan Disability Scale. Int Clin Psychopharmacol. 2008 Mar;23(2):70-83.

17. Schwartz RC. Concurrent validity of the Global Assessment of Functioning Scale for clients with Schizophrenia. Psychol Rep. 2007 Apr;100(2):571-4.

18. Meoni P, Salinas E, Brault Y, Hacket D. Pattern of symptom improvement following treatment with venlafaxin XR in patients with generalized anxiety disorder. J Clin Psychiatry. 2001 Nov;62(11):888-93.

19. Hamilton M. A rating scale for depression. J Neurol Neurosurg Psychiat. 1960 Feb;23:56-62.

20. Bech P. Rating Scales for Psychopathology, Health Status and Quality of Life. A Compendium on Documentation in Accordance with the DSM-III-R and WHO Systems. 1 ed. Berlin: Springer Verlag; 1993.

21. Kiresuk TJ, Sherman RE. Goal attainment scaling: a general method of evaluating comprehensive community mental health programs. Community Mental Health Journal 1968 Dec;4:443-53.

22. Lehrer PM, Woolfolk RL, Sime WE, Barlow DH. Principles and Practice of Stress Management. 3rd ed. New York: Guilford Press; 2007.

23. Lazarus RS, Folkman S. Stress Appraisal and Coping. New York: Springer Publishing; 1984.

24. Meichenbaum D. Stress Inoculation Training, a Preventive and Treatment Approach. In: Lehrer PM, Woolfolk RL, Sime WE, editors. Principles and Practice of Stress Management. 3rd ed. New York: Guilford Press; 2007. p. 497-518.

25. Bernstein DA, Carlson RC, Schmidt JE. Progressive Relaxation: Abbreviated methods. In: Lehrer PM, Woolfolk RL, Sime WE, editors. Principles and Practice of Stress Management. 3rd ed. New York: Guilford Press; 2007. p. 88-122.

26. Giardino ND, McGrady A, Andrasik F. Stress Management and Relaxation Therapies for Somatic Disorders. In: Lehrer PM, Woolfolk RL, Sime WE, editors. Principles and Practice of Stress Management. 3rd ed. New York: Guilford Press; 2007. p. 682-702. 